\section{IMMOBILIZED ENZYMES}

\section{Enhancing DNA sequencing}

Bioconjug. Chem. 28, 2077-2085 (2018).

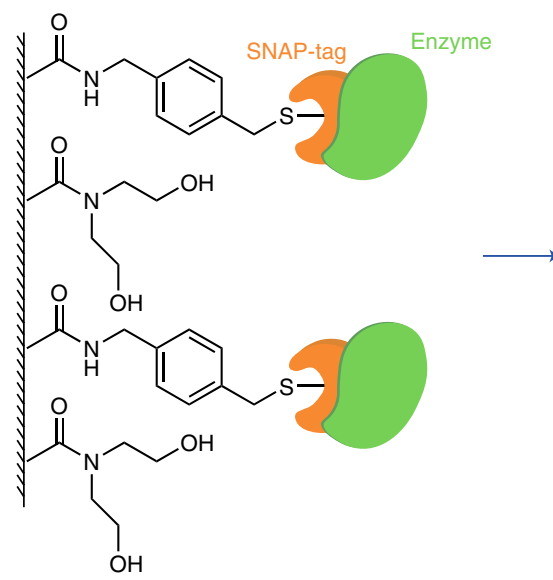

Credit: American Chemical Society

Next-generation sequencing (NGS) revolutionized the biological sciences and genomic research in particular by providing a fast and scalable ultra-high throughput method. Hereby, genomes are usually fragmented into small pieces to facilitate their sequencing in a single experimental setup. The ends of the fragments need to be repaired, whereby T4 DNA polymerase (T4 pol) and T4 polynucleotide kinase (T4 PNK) are typically used. For the subsequent DNA modification step, these enzymes are generally heat inactivated, which is assumed to negatively affect the sequencing of AT-rich regions.

Now, Xu and co-workers from the New England Biolabs Inc. avoid the heat inactivation step by immobilizing T4 pol and T4 PNK on magnetic beads allowing their separation from the reaction mixture. Enzymes expressed with a SNAP-Tag were covalently attached to the functionalized surface of the beads. Initially, unfavourable interactions with the surface were indicated by a reduced exonuclease and polymerase activity of immobilized T4 pol limiting the yield in a model reaction to $70 \%$. Therefore, flexible PEG-chains were introduced on the bead-surface rendering the enzyme environment less hydrophobic. This significantly improved the activity of immobilized T4 pol restoring full conversion
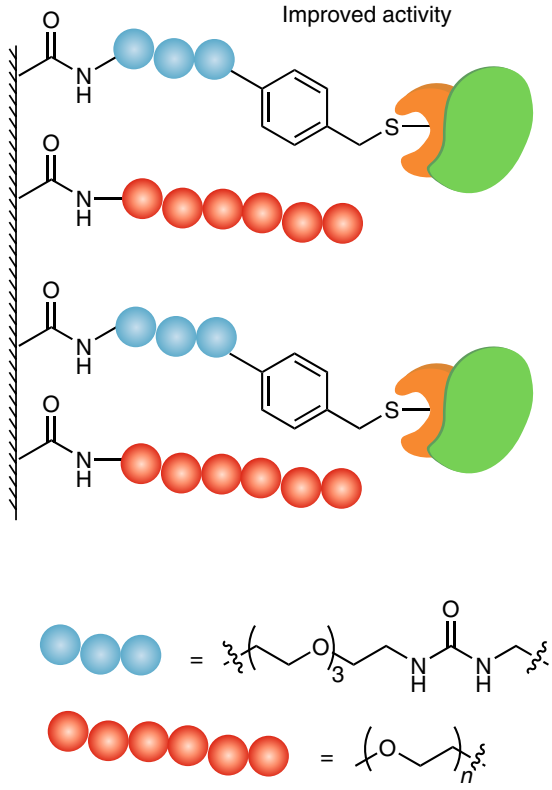

of the DNA substrates, while increasing the thermostability of the enzyme.

T4 pol kinetic studies indicated that PEG-coating increased the nucleotide incorporation rate by more than 100 -fold while also enhancing DNA dissociation. This enabled successful application of immobilized T4 pol and T4PNK in the Illumina sequencing platform while avoiding heat-inactivation by magnetic separation. The DNA library yields, percentage of unmapped reads, chimeras and mismatched reads were competitive to those of soluble enzymes. Remarkably, the read coverage in the high AT-content region was significantly improved for the immobilized enzymes relative to that of soluble enzymes.

The results of this study indicate that immobilized enzymes may have potential use in NGS library construction. Further, other researchers aiming at the application of immobilized enzymes in the red-biotechnology sector might benefit from the reported effects of the surface modification on the kinetics of the DNA processing enzymes.

Jan-Stefan Völler

Published online: 12 July 2018

https://doi.org/10.1038/s41929-018-0120-7 